Research Paper

\title{
Endometrial Cancer Incidence in Breast Cancer Patients Correlating with Age and Duration of Tamoxifen Use: a Population Based Study
}

\author{
Ju-Yin Chen 1,2, Shou-Jen Kuo², Yung-Po Liaw³, Itzhak Avital"4, Alexander Stojadinovic', Yan-gao Man', \\ Ciaran Mannion" ${ }^{5}$, Jianlian Wang, ${ }^{6}$, Ming-Chih Chou ${ }^{7,8}$, Horng-Der Tsai ${ }^{9}$, Shou-Tung Chen ${ }^{2}$, Yi-Hsuan \\ $\mathrm{Hsiao}^{7,9}{ }^{凶}$ \\ 1. Department of Epidemiology, School of Public Health, University of California Los Angeles, USA. \\ 2. Comprehensive Breast Cancer Center, Changhua Christian Hospital, Changhua, Taiwan. \\ 3. Department of Public Health and Institute of Public Health, Chung Shan Medical University, Taichung, Taiwan. \\ 4. Bon Secours Cancer Institute, Bon Secours Health System, Richmond, VA, USA. \\ 5. Department of Pathology, Hackensack University Medical Center, Hackensack, NJ, USA. \\ 6. Lombardi Comprehensive Cancer Center, Georgetown University, Washington, DC, USA. \\ 7. School of Medicine, Chung Shan Medical University, Taichung, Taiwan. \\ 8. Division of Thoracic Surgery, Department of Surgery, Chung Shan Medical University Hospital, Taichung, Taiwan. \\ 9. Department of Obstetrics and Gynecology, Changhua Christian Hospital, Changhua, Taiwan.
}

$\square$ Corresponding author: Yi-Hsuan Hsiao, M.D., PhD, Assistant Professor, School of Medicine, Chung Shan Medical University, Department of Obstetrics and Gynecology, Changhua Christian Hospital, 135 Nanhsiao Street, Changhua, 500 Taiwan. Phone: 886-4-7238595; Fax: 886-4-7228289; E-mail: 54315@cch.org.tw. Or Shou-Tung Chen, M.D. Assistant Professor, Comprehensive Breast Cancer Center, Changhua Christian Hospital, 135 Nanhsiao Street, Changhua, 500 Taiwan. Phone: 886-4-7238595 ; Fax: 886-4-7228289 ; E-mail: 1886@cch.org.tw

(c) Ivyspring International Publisher. This is an open-access article distributed under the terms of the Creative Commons License (http://creativecommons.org/ licenses/by-nc-nd/3.0/). Reproduction is permitted for personal, noncommercial use, provided that the article is in whole, unmodified, and properly cited.

Received: 2013.12.21; Accepted: 2014.01.12; Published: 2014.01.23

\begin{abstract}
Background: Our study aimed to assess the endometrial cancer risk after tamoxifen adjuvant treatment for female breast cancer patients in Taiwan.

Materials and Methods: A total of 74,280 breast cancer patients between January 1997 and December 2004 were included in the study; 39,4II received tamoxifen treatment and 34,869 did not. Tamoxifen-associated endometrial cancer was defined as endometrial cancer that occurred in patients at least 6-month after the diagnosis of breast cancer, who underwent tamoxifen treatment.

Results: A total of 222 patients developed endometrial cancer, and of these, $153(69 \%)$ were seen in patients with tamoxifen treatment, and $69(31 \%)$ were seen in patients without the use of tamoxifen. The incidence of endometrial cancer was $0.388 \%(153 / 39,4 \mathrm{II})$ in patients with tamoxifen treatment, while was $0.198 \%(69 / 34,869)$ in patients without tamoxifen treatment. Logistic regression analysis demonstrated that tamoxifen use and age over 35 years were significantly correlated with development of endometrial cancer $(p<0.00 \mathrm{I}$ and $p=0.002$, respectively). The odds ratio was $2.94(95 \% \mathrm{Cl}, 2.13-4.06)$ for 3 years or longer tamoxifen use. The odds ratio was $4.08(95 \% \mathrm{Cl}, 1.67-9.93)$ for women older than 35 years compared to those 35 or younger than 35 years. There were no significant differences in prior hormone exposure, hypertension and diabetes.

Conclusions: To the best of our knowledge, this is the largest population based study that shows in patients with breast cancer, tamoxifen use for more than three years or patients older than 35 years was associated with a significantly increased risk for developing endometrial cancer.
\end{abstract}

Key words: breast cancer, tamoxifen, endometrial cancer 


\section{Introduction}

Tamoxifen is an effective treatment in reducing recurrence and mortality rates for estrogen receptor (ER) positive breast cancer patients (1). On the other hand, an undesirable side effect of increasing risk of endometrial cancer has been suspected for decades (2-6). A number of case-control studies showed that the risk of endometrial cancer increased after long-term tamoxifen treatment for breast cancer patients (2-6). A vast majority of these case-control studies were conducted in the western world with a small sample size (from a total of a few hundred to a few thousand cases in each study (2-6). The data of tamoxifen use from Asia countries were scarce. A previous study of 674 consecutive breast patients in Japan reported that tamoxifen use for longer than 5 years correlated with increasing risk of developing endometrial cancer, while the small sample size prevented to detect statistical significance (7).

A more recent study analyzing pooled studies from the Cochrane Central Register of Controlled Trials and National Library of Medicine for published data from January 1970 to December 2010, however, reported that "The risk of endometrial cancer, deep vein thrombosis and pulmonary embolism is low in women $<50$ years who take tamoxifen for breast cancer prevention. The risk decreases from the active to follow-up phase of treatment (8). In addition, although levonorgestrel intrauterine system (LNG-IUS) has been used in patients with breast patients taking tamoxifen (TAM) to prevent endometrial proliferation, the benefits, the side effects, and the patients suitable for this treatment are still elusive (9).

The above facts clearly indicate the needs for further clarification of the potential link between Tamoxifen use and the risk for endometrial cancer. Since the incidences of breast and endometrial cancer have been increasing (Figure 1), according to the Taiwan cancer registration system (https://cris.bhp. doh.gov.tw/pagepub/Home.aspx), our study aimed to assess the endometrial cancer risk after tamoxifen adjuvant treatment for female breast cancer patients

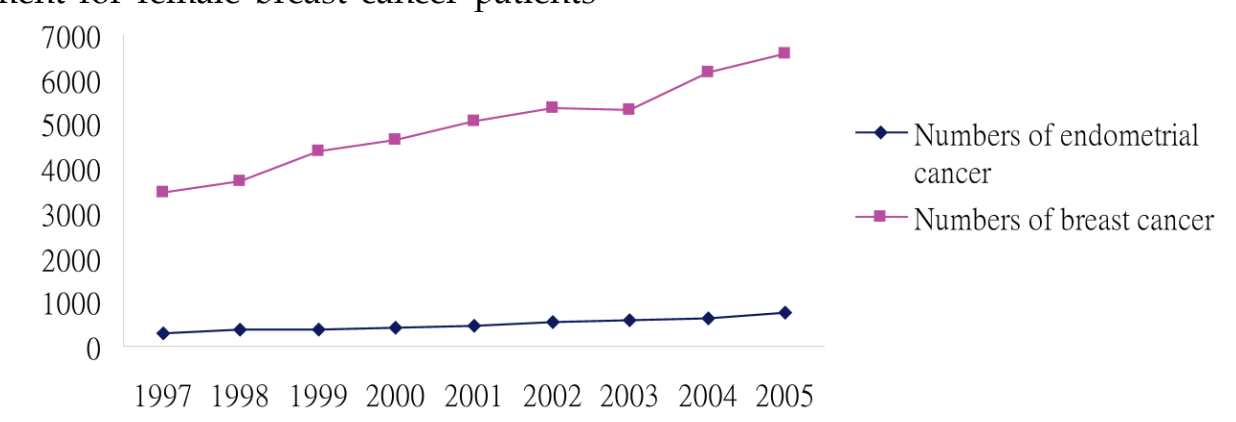

Figure I. Trends in endometrial cancer and breast cancer in Taiwan during 1997 through 2005. Data sources: Taiwan cancer registration system. in Taiwan using a significantly large and population-based database.

\section{Materials and Methods}

A retrospective cohort study was conducted to evaluate tamoxifen use on endometrial cancer risk for breast cancer patients in Taiwan. In our study, the data included details of inpatient orders files, inpatient expenditures by admissions files, details of ambulatory care orders files and ambulatory care expenditures files. The above data were obtained from the National Health Insurance Research Database (NHIRD) (http://www.nhri.org.tw/nhird/en/index. htm) provided by the Bureau of National Health Insurance, Department of Health, and managed by National Health Research Institutes. All identifiers of all patients were completely removed before the release of the documentation and the encrypted data used in our study cannot link to any of the specific individual.

A total of 74,280 patients treated for breast cancer between January 1, 1997 and December 31, 2004 were included in the study. The cases were identified according to the coding of Ninth Revision International Classification of Diseases (ICD-9) 1740-1749 (malignant neoplasm of female breast). Of the 74,280 patients, 39,411 (53.1\%) received tamoxifen treatment, and $34,869(46.9 \%)$ did not. The duration of tamoxifen treatment was timed from the first to the last prescription for tamoxifen. Tamoxifen-associated endometrial cancer was defined as endometrial cancer that occurred in patients at least 6-month after the diagnosis of breast cancer, who underwent tamoxifen treatment.

Descriptive statistics were used for patients' demographic characteristics analysis. Logistic regression analysis was performed to assess the potential association between the development of endometrial cancer with tamoxifene treatment, prior hormone exposure, hypertension, diabetes and age. The SAS statistical package (SAS Institute Inc., Cary, NC. Version 9.1) was used to perform analysis. The statistical significance was adopted at $p$ values of less than 0.05 . 


\section{Results}

A total of 222 patients developed endometrial cancer. The characteristics of these 222 patients are shown in Table 1. Of the 222 endometrial cancer cases, 153 (69 \%) were seen in patients with tamoxifen treatment, and $69(31 \%)$ were seen in patients without the use of tamoxifen. The incidence of endometrial cancer was $0.388 \%(153 / 39,411)$ in patients with tamoxifen treatment, while was $0.198 \%(69 / 34,869)$ in patients without tamoxifen treatment $(\mathrm{p}<0.001)$.

Among patients with tamoxifen treatment, the average duration of tamoxifen use was 2.91 years in patients developed endometrial cancer, and was 1.67 years in patients did not develop endometrial cancer (Table 2).

Table I. Characteristics of 222 breast cancer patients with endometrial cancer in the non tamoxifen and tamoxifen groups

\begin{tabular}{|c|c|c|c|}
\hline Characteristics & $\begin{array}{l}\text { Numbers }(\%) \text { of } \\
\text { Endometrial can- } \\
\text { cer cases with } \\
\text { Tamoxifen Use }\end{array}$ & $\begin{array}{l}\text { Numbers }(\%) \text { of } \\
\text { Endometrial cancer } \\
\text { cases without Ta- } \\
\text { moxifen Use }\end{array}$ & $\begin{array}{l}P \\
\text { value }\end{array}$ \\
\hline Total & $153(100)$ & $69(100)$ & \\
\hline Age & & & 0.96 \\
\hline$<=35$ & $4(2.6)$ & $1(1.45)$ & \\
\hline$>35$ & $149(97.39)$ & 68 (98.55) & \\
\hline Prior Diabetes & & & 0.37 \\
\hline Yes & $7(4.58)$ & $6(8.7)$ & \\
\hline No & $146(95.42)$ & $63(91.30)$ & \\
\hline Prior Hypertension & & & 0.80 \\
\hline Yes & $19(12.42)$ & $7(89.86)$ & \\
\hline No & $134(87.58)$ & $62(10.14)$ & \\
\hline Prior Hormone & & & \\
\hline Exposure & & & 0.14 \\
\hline Yes & 82 (53.59) & $45(65.22)$ & \\
\hline No & $71(46.41)$ & $24(34.78)$ & \\
\hline $\begin{array}{l}\text { Time between the } \\
\text { Recruitment and } \\
\text { diagnosis of En- } \\
\text { dometrial Cancer }\end{array}$ & & & 0.20 \\
\hline $\begin{array}{l}6 \text { months } \sim \leqq 1.5 \\
\text { year }\end{array}$ & $42(27.45)$ & $28(40.58)$ & \\
\hline $1.5 \sim \leqq 3$ years & 45 (29.41) & $20(28.99)$ & \\
\hline $3 \sim \leqq 4.5$ years & $32(20.92)$ & 12 (17.39) & \\
\hline $4.5 \sim \leqq 6$ years & $25(16.34)$ & $8(11.59)$ & \\
\hline$>6$ years & $9(5.88)$ & $1(1.45)$ & \\
\hline
\end{tabular}

Table 2. Durations of tamoxifen treatment for $39,41 \mathrm{I}$ breast cancer patients

\begin{tabular}{lll}
\hline Patient status & $\begin{array}{l}\text { Number of } \\
\text { patients }\end{array}$ & $\begin{array}{l}\text { Average dura- } \\
\text { tion (years) }\end{array}$ \\
\hline With endometrial cancer & 153 & 2.91 \\
Without endometrial cancer & 38,258 & 1.67 \\
\hline
\end{tabular}

Logistic regression of endometrial cancer risk for breast cancer patients on prior hormone exposure, hypertension, diabetes, age and tamoxifene treatment are shown in Table 3. Tamoxifen use and age over 35 years were significantly correlated with the development of endometrial cancer $(p<0.001$ and $p=0.002$, respectively). Patients treated with tamoxifen had a significantly higher risk of endometrial cancer than those who did not take tamoxifen (OR 1.86; 95\%CI, $1.40-2.47$; $\mathrm{p}$ 0.001). However, there were no significant differences in prior hormone exposure, hypertension and diabetes.

Logistic regression of endometrial cancer risk for breast cancer patients with tamoxifen use on duration of tamoxifen treatment, prior hormone exposure, hypertension, diabetes and age are shown in Table 4. A link between long-term tamoxifen use and increasing endometrial cancer risk by odds ratio was 2.94 (95\% CI, 2.13-4.06) for 3 years or longer; the odds ratio was $4.08(95 \% \mathrm{CI}, 1.67-9.93)$ for women aged older than 35 years compared to those aged 35 or younger than 35 years for breast cancer patients with tamoxifen use. There were no significant differences in prior hormone exposure, hypertension and diabetes.

Table 3. Logistic regression of endometrial cancer risk for 74,280 breast cancer patients on prior hormone exposure, hypertension, diabetes, age and tamoxifene treatment

\begin{tabular}{llllll}
\hline & \multirow{2}{*}{ OR } & & \multicolumn{2}{l}{$\mathbf{9 5 \%} \mathbf{C I}$} & p-value \\
\cline { 5 - 7 } & & & Lower & Upper & \\
\hline Tamoxifen use & 1.86 & & 1.40 & 2.47 & $<0.001$ \\
Age above 35 years old & 4.16 & & 1.71 & 10.14 & 0.002 \\
Prior hormone exposure & 0.99 & & 0.75 & 1.29 & 0.92 \\
Prior hypertension & 0.71 & 0.47 & 1.08 & 0.11 \\
Prior diabetes & 1.14 & 0.64 & 2.02 & 0.66 \\
\hline
\end{tabular}

Table 4. Logistic regression of endometrial cancer risk for 39,4I I breast cancer patients with tamoxifen use on duration of tamoxifen treatment, prior hormone exposure, hypertension, diabetes and age

\begin{tabular}{|c|c|c|c|c|}
\hline & OR & $95 \% \mathrm{CI}$ & & p-value \\
\hline & & Lower & Upper & \\
\hline Less than 3 years tamoxifen use & 1.32 & 0.95 & 1.83 & 0.10 \\
\hline 3 years or above of tamoxifen use & 2.94 & 2.13 & 4.06 & $<0.001$ \\
\hline Age above 35 years old & 4.08 & 1.67 & 9.93 & 0.002 \\
\hline Prior hormone exposure & 1.00 & 0.76 & 1.31 & 0.10 \\
\hline Prior hypertension & 0.74 & 0.49 & 1.13 & 0.17 \\
\hline Prior diabetes & 1.17 & 0.66 & 2.07 & 0.60 \\
\hline
\end{tabular}

\section{Discussion}

To the best of our knowledge, our study is the largest population based study to further assess the potential association between tamoxifen use for breast cancer treatment and the risk for endometrial cancer. 
Our study reveals that tamoxifen use and age over 35 years were significantly correlated with the development of endometrial cancer for breast cancer patients. Furthermore, for breast cancer patients undergoing tamoxifen treatment, a link between long-term tamoxifen use and increasing endometrial cancer risk by odds ratio was 2.94 (95\% CI, 2.13-4.06) for 3 years or longer; the odds ratio was 4.08 (95\% CI, 1.67-9.93) for women aged older than 35 years compared to those aged 35 or younger than 35 years. There were no significant differences in prior hormone exposure, hypertension and diabetes.

Our findings are largely in line with those of previous studies. A case-control study revealed a significantly increasing endometrial cancer risk by $\mathrm{OR}$ 3.6 (95\% CI; 2.6 to 4.8 ) for breast cancer women with tamoxifen treatment beyond five years compared those women with no treatment (4). The Comprehensive Cancer Centres' ALERT Group detected an increasing endometrial cancer risk for longer duration of tamoxifen use, relative risk 6.9 (95\%CI, 2.4-19.4) for at least 5 years and $2.0(95 \% \mathrm{CI}, 1.2-3.2)$ for $2-5$ years compared with non-users in breast cancer women (6).

Our study has also obtained several unique findings that are not sufficiently addressed in previous studies, such the duration of tamoxifen use and patients' age. Previous studies showed an increasing endometrial cancer risk after 5-year tamoxifen use (4, 6), while our study reveals the risk impact can occur fewer than 3-years. Our study has suggested that age is an important factor for the development of endometrial cancer in breast cancer patients. In our study, an increasing endometrial cancer risk by OR 4.16 (95\% $\mathrm{CI}=1.71$ to 10.14 ) was observed in the breast cancer women aged older than 35 years compared with the younger breast cancer patients (Table 3). Our study has also detected an increasing endometrial cancer risk by OR 4.08 (95\% CI = 1.67 to 9.93) in breast cancer patients with tamoxifen treatment for women older than 35 years compared with the younger ones (Table $4)$.

It is very important to prevent endometrial cancer developing or diagnose at early stage as tamoxifen is commonly used for all stages of hormone receptor positive breast cancer patients. A previous study reported that the endometrium thickness cumulatively increased during the tamoxifen treatment and the thicker endometrium remained years after the end of treatment, which results in increasing endometrial cancer development (10). Another study showed that breast cancer patients with tamoxifen use have early stage endometrial cancer and good prognoses (11). The incidence of subsequent endometrial carcinoma does not increase for primary breast cancer patients with tamoxifen usage if the patients under- went annual screening for gynecologic malignancies, including a pelvic examination, cervix and endometrium examination (12). Regular follow-up of the endometrium thickness in breast cancer women taking tamoxifen may play an important role for endometrial carcinoma early diagnosis (10).

The risk of endometrial cancer has been traditionally reported for postmenopausal women receiving long term tamoxifen therapy. However, more than half of patients in this study appear to have developed cancer within 3 years, almost a third within 18 months. It was different from the western countries. The incidence peak of Taiwanese female breast cancer is also different form the western countries (13). The clinical implications need more studies for evaluating the pathogenesis, environmental genetics and other factors.

\section{Conclusions}

Our study shows that tamoxifen use and age over 35 years significantly correlated with breast cancer patients with endometrial cancer. An increased awareness among gynecologic health provider may help early diagnose endometrial cancer. Regular gynecologic follow-up may be mandatory for those women with three years or above of tamoxifen use and age over 35 years.

\section{Acknowledgments}

This study is based in part on data from the National Health Insurance Research Database provided by the Bureau of National Health Insurance, Department of Health and managed by National Health Research Institutes. The interpretation and conclusions contained herein do not represent those of Bureau of National Health Insurance, Department of Health or National Health Research Institutes.

\section{Competing Interests}

The authors have declared that no competing interest exists.

\section{References}

1. Early Breast Cancer Trialists' Collaborative Group (EBCTCG), Davies C,Godwin J, Gray R, Clarke M, Cutter D, Darby S, McGale P, Pan HC, Taylor C,Wang YC, Dowsett M, Ingle J, Peto R: Relevance of breast cancer hormone receptors and other factors to the efficacy of adjuvant tamoxifen: patient-level meta-analysis of randomised trials. Lancet 2011; 378:771-784.

2. van Leeuwen FE, Benraadt J, Coebergh JW, Kiemeney LA, Gimbrere CH, Otter R, Schouten LJ, Damhuis RA, Bontenbal M, Diepenhorst FW, et al. Risk of endometrial cancer after tamoxifen treatment of breast cancer. Lancet 1994; 343:448-452.

3. Bernstein L, Deapen D, Cerhan JR, Schwartz SM, Liff J, McGann-Maloney E, Perlman JA, Ford L: Tamoxifen therapy for breast cancer and endometrial cancer risk. J Natl Cancer Inst 1999; 91:1654-1662.

4. Swerdlow AJ, Jones ME: Tamoxifen treatment for breast cancer and risk of endometrial cancer: a case-control study. J Natl Cancer Inst 2005; 97:375-384.

5. Endometrial cancer survival after breast cancer in relation to tamoxifen treatment: Pooled results from three countries Jones et al. Breast Cancer Research 2012; 14:R91 
6. Bergman L, Beelen ML, Gallee MP, Hollema H, Benraadt J, van Leeuwen FE: Risk and prognosis of endometrial cancer after tamoxifen for breast cancer. Comprehensive Cancer Centres' ALERT Group. Assessment of Liver and Endometrial cancer Risk following Tamoxifen. Lancet 2000; 356:881-887.

7. Yamazawa K, Miyazawa Y, Suzuki M, Wakabayashi M, Kaku H, Matsui H, Sekiya S. Tamoxifen and the risk of endometrial cancer in Japanese women with breast cancer. Surg Today. 2006; 36(1):41-6.

8. Iqbal J, Ginsburg OM, Wijeratne TD, Howell A, Evans G, Sestak I, Narod SA. Endometrial cancer and venous thromboembolism in women under age 50 who take tamoxifen for prevention of breast cancer: a systematic review. Cancer Treat Rev. 2012; 38(4):318-28.

9. Gizzo S, Di Gangi S, Bertocco A, Noventa M, Fagherazzi S, Ancona E, Saccardi C, Patrelli TS, D'Antona D, Nardelli GB. Levonorgestrel Intrauterine System in Adjuvant Tamoxifen Treatment: Balance of Breast Risks and Endometrial Benefits--Systematic Review of Literature. Reprod Sci. 2013; [Epub ahead of print]

10. Lindahl B, Andolf E, Ingvar C, Ranstam J, Willén R. Adjuvant tamoxifen in breast cancer patients affects the endometrium by time, an effect remaining years after end of treatment and results in an increased frequency of endometrial carcinoma. Anticancer Res. 2008; $28: 1259-62$.

11. Behtash N, Hashemi R, Karimi Zarchi M. Uterine malignancy following tamoxifen use in breast cancer patients in Iran: case series and literature review. Asian Pac J Cancer Prev. 2009; 10(1):163-6.

12. Katase K, Sugiyama Y, Hasumi K, Yoshimoto M, Kasumi F. The incidence of subsequent endometrial carcinoma with tamoxifen use in patients with primary breast carcinoma. Cancer. 1998; 82(9):1698-703.

13. Ho ML, Liaw YP, Lai CH, Chen YY, Tsai HD, Chou MC, Hsiao YH. Significantly increased medical expenditure on breast cancer failing to bring down its mortality and incidence rate. J Cancer. 2013;4(7):531-5 